\title{
Hospital Antibiogram- A Tool for Safe Antimicrobial Therapy
}

\author{
Rekha Rai1, Pratibha Bhat ${ }^{2}$ \\ ${ }^{1}$ Department of Microbiology, KS Hegde Medical Academy, Mangaluru, Karnataka, India. \\ ${ }^{2}$ Department of Microbiology, KS Hegde Medical Academy, Mangaluru, Karnataka, India.
}

\section{ABSTRACT}

\section{BACKGROUND}

Monitoring emerging trends in antimicrobial resistance at local levels is a very important aspect for clinical decision making and infection control interventions in this era of rising superbugs. The hospital antibiogram constructed by standardized methods is a summary of antimicrobial susceptibilities of local bacterial isolates, with periodic review.

\section{METHODS}

This antibiogram was prepared based on the Clinical and Laboratory Standards Institute (CLSI) M39 - A4 guidelines. The data was collected for a period of three years from May 2016 to April 2019. All the samples which were received in the microbiology laboratory for aerobic bacterial culture and sensitivity testing were considered. Antibiotic susceptibility data of the bacterial isolates processed by VITEK2 system was considered. A hospital antibiotic policy was formulated and implemented.

\section{RESULTS}

A total of 15,135 samples were analysed from different clinical departments for a period of three years, of which 5,638 were culture positive. A comparative analysis of the three years' data during the introduction of antibiotic protocol showed a gradual decrease in the rate of infection with multidrug resistant organisms. The prevalence of Methicillin Resistant Staphylococcus aureus (MRSA) showed a decrease from $15 \%$ to $7 \%$. Extended spectrum beta-lactamase (ESBL) producing Escherichia coli in urine decreased from $42.4 \%$ to $27 \%$.

\section{CONCLUSIONS}

Prompt antimicrobial therapy in case of an infection makes a lot of difference between recovery and death and most of the time prevents long term disability. Hence, antibiotic policy is one of the mandatory requirements, and making an antibiogram is the first step before framing antibiotic policy.

\section{KEY WORDS}

Antibiogram, Drug Resistance, Antibiotics
Corresponding Author: Dr. Pratibha Bhat, Assistant Professor, Department of Microbiology, KS Hegde Medical Academy, Mangaluru-575018, Karnataka, India. E-mail: pratibhabhat.u@gmail.com

DOI: $10.14260 /$ jemds/2020/28

Financial or Other Competing Interests: None.

How to Cite This Article:

Rai R, Bhat P. Hospital Antibiogram- A Tool for Safe Antimicrobial Therapy. J. Evolution Med. Dent. Sci. 2020;9(03):124128, DOI: $10.14260 /$ jemds/2020/28

Submission 12-10-2019,

Peer Review 31-12-2019,

Acceptance 06-01-2020,

Published 20-01-2020. 


\section{BACKGROUND}

It has been estimated that by 2050, 10 million deaths annually will be due to antimicrobial resistance (AMR), which is emerging as a major threat to public health. ${ }^{1}$ India is said to carry the largest burden of drug-resistant pathogens worldwide. New Delhi Metallo- $\beta$-lactamase (NDM-1) reported in 2008, which showed a rapid spread to other countries was named after India's capital.2 India has the highest burden of bacterial diseases in the world. Hence, antibiotics play a major role in healthcare with India being one of the largest consumers of antibiotics worldwide. The 2015 World Health Organization (WHO) survey on public understanding of antibiotic usage and resistance revealed that $76 \%$ of the respondents from India reported having taken antibiotics in the past 6 months. Also, $75 \%$ of the respondents believed that cold and flu could be treated with antibiotics. ${ }^{3}$

Development of mechanisms by the microorganisms to evade antimicrobial action leads to development of AMR. Misuse and overuse of antibiotics are the major factors contributing to AMR. Hence, monitoring of antimicrobial resistance trends is essential at each health care center. It plays a key role at the bedside, in deciding the antimicrobial therapy a patient receives. The knowledge of the local antimicrobial resistance patterns is also vital to introduce appropriate infection control measures to check the spread of these resistant organisms as well as to prevent the emergence of new drug resistant bugs.

A cumulative antibiogram report is an annual summary of susceptibility rates of the commonly isolated organisms in a health care setup. This report helps in monitoring the trends of antimicrobial resistance. ${ }^{4}$ The hospital antibiogram which is constructed by standardized methods is a summary of antimicrobial susceptibilities of local bacterial isolates, with periodic review. The most frequent use of cumulative antibiogram report is in guiding initial empirical antimicrobial therapy decisions for the management of infections in patients for whom microbiological test data do not exist. 4,5

The Clinical and Laboratory Standards Institute (CLSI) has developed guidelines, namely CLSI M39 - A4 guidelines for formulation of cumulative antimicrobial susceptibility test data by health care facilities. ${ }^{6}$ CLSI gives practical recommendations for analysis and presentation of antimicrobial susceptibility trends. The results derived in accordance with the guideline would be comparable between institutions. It also overcomes the parameters which tend to over-estimate drug resistance rates like not including surveillance isolates and including the first isolate per patient during the period of analysis. ${ }^{4}$

CLSI M39-A4 gives a detailed description to persons involved in the preparation and use of cumulative antibiogram reports, as well as for information technology managers who are involved in designing and supporting the clinical laboratory's data management. The document contains specific recommendations for the collection, storage, analysis, and presentation of data and includes sample templates that highlight the recommendations. Critical issues addressed include the recommended frequency of reporting, the number of isolates to include in a statistic, and a mechanism for eliminating multiple isolates of a given bacterial species obtained from an individual patient. ${ }^{6}$

\section{METHODS}

This is a prospective observational study conducted in a tertiary care hospital in South India. The study was approved by the institutional ethics committee. The antibiogram was prepared by the microbiology laboratory of the hospital, based on the CLSI M39 - A4 guidelines. ${ }^{6}$ The data was collected for a period of three years from May 2016 to April 2019. The antibiogram was prepared for each year. All the samples which were received in the microbiology laboratory for aerobic bacterial culture and sensitivity testing were considered. The bacterial isolates from diagnostic clinical samples were subjected to VITEK2 system analysis for the detection of minimum inhibitory concentration (MIC) values. The first isolate per patient in the period analysed, irrespective of the body site from which the specimen was obtained or the antimicrobial susceptibility pattern was considered. Isolates from surveillance and screening cultures were excluded. Repeat isolates were not considered. In accordance with CLSI M39-A46 recommendations all the antimicrobials which were routinely tested for an isolate were analysed for cumulative antibiogram preparation. The percentage of isolates that were susceptible only was included. The percentage of isolates with intermediate susceptibility was excluded.

Based on the above parameters, an institution antibiotic policy was formulated and implemented. At the end of one year of implementation of the antibiotic policy a random prescription audit was conducted amongst inpatient and outpatient departments. The following parameters were evaluated i. Choice of empiric antibiotic therapy ii. Route of administration iii. The dose and the duration of treatment. In case of non-compliance documentation of the reason for the same was sought.

\section{Statistical Analysis}

The data was analysed and expressed using descriptive statistics such as counts and percentage. The significance of the data was determined using $2 \times 2$ contingency table and Fisher exact test.

\section{RESULTS}

A total of 15,135 samples were analysed from different clinical departments for a period of three years of which; 5,638 were culture positive. The distribution of growth in culture positive samples is shown in table 1. Initially, during the first year (May 2016-April 2017), the antibiogram for individual isolates was formulated based on gram reaction, genus and species level identification and type of sample and their susceptibility pattern. This gave an insight into the common isolates and their susceptibility patterns. An initial presentation of the results based on organisms and their susceptibility patterns found partial acceptance amongst the clinicians, which changed to better acceptance when changed as per site of infection. For example, the common isolates 
from urinary tract infections and the susceptibility profile of the most common isolate as presented to the clinicians is shown in figure 1 and figure 2 . In the same manner, the common isolates from skin and soft tissue infections, respiratory infections, blood stream infections were analysed and the susceptibility profile of each of the isolates was depicted graphically.

Based on these results, an antibiotic policy was formulated through the antibiotic policy committee which included the medical superintendent, representations from clinical departments, clinical microbiologist, pharmacy, infection control officer. The antibiotic policy implemented at our hospital emphasized 4 general rules for antibiotic prescribing namely; i. All antibiotic initiations should be done after sending appropriate cultures, ii. Follow the hospital policy when choosing antimicrobial therapy wherever possible, iii. Change in antibiotic would be done after sending fresh cultures, iv. If alternative antibiotics are chosen to document the reason in the case records. The policy included the antibiogram of the previous year and the empiric antibiotic therapy recommended for various infections like skin and soft tissue, urinary and respiratory infections. Empiric therapy for bloodstream infections was not formulated as the predominant isolate as Coagulase negative Staphylococcus, which depicted improper blood sampling for bacterial culture. The policy also included the standard dosage of the recommended antibiotics. The policy was formulated based on the hospital cumulative antibiogram and the national treatment guidelines for antimicrobial use in infectious diseases. ${ }^{7}$ A prescription audit performed amongst inpatient and outpatient departments showed a good compliance amongst the treating clinicians. The audit showed $60 \%$ compliance from the treating physicians.

A comparative analysis of the three years' data in the process of introduction of antibiotic protocol showed a gradual decrease in the rate of infection with multidrug resistant organisms as shown in table 2. The prevalence of Methicillin Resistant Staphylococcus aureus (MRSA) showed a decrease from $15 \%$ to $7 \%$. Extended spectrum betalactamase (ESBL) producing Escherichia coli in urine decreased from $42.4 \%$ to $27 \%$.

Percentage of isolates susceptible to various antibiotics before (2016-2017 data) and 2 years after implementation of the antibiotic policy (2017 to 2019 data) is depicted in table 3 . A significant change (p value) was noted among $E$. coli and Klebsiella isolates from exudates samples and Pseudomonas isolates from sputum samples. A rise in the susceptibility percentage was seen among isolates from exudates samples. Isolates from blood showed a fall in the susceptibility percentage towards various antibiotics, though the change was not statistically significant. The analysis of MIC values showed that some of the isolates had lower MIC values after implementation of the policy; for example, the MIC values of E. coli for the antibiotic amikacin is shown in table 4 .

\begin{tabular}{|c|c|c|c|}
\hline Urine (n=1704) & Exudates (n=2816) & Sputum(n=399) & Blood (n=719) \\
\hline & & & S. aureus $=71$ \\
E. coli $=798$ & S. aureus $=423$ & & Coagulase negative \\
Klebsiella $=345$ & Pseudomonas $=413$ & Klebsiella $=127$ & Staphylococcus \\
Pseudomonas $=97$ & E. coli $=310$ & Pseudomonas $=49$ & $=373$ \\
Enterococcus $=163$ & Klebsiella $=415$ & Acinetobacter $=50$ & E. coli $=57$ \\
Others $=301$ & Enterococcus $=103$ & Others $=173$ & Klebsiella $=59$ \\
& Others $=1152$ & & Acinetobacter $=34$ \\
& & & Others $=125$ \\
\hline
\end{tabular}

\begin{tabular}{|c|c|c|c|}
\hline $\begin{array}{c}\text { Common } \\
\text { Pathogens }\end{array}$ & $\begin{array}{c}\text { Prevalence } \\
\mathbf{2 0 1 6}-\mathbf{2 0 1 7}\end{array}$ & $\begin{array}{c}\text { Prevalence } \\
\mathbf{2 0 1 7}-\mathbf{2 0 1 8}\end{array}$ & $\begin{array}{c}\text { Prevalence } \\
\mathbf{2 0 1 8} \text { - 2019 }\end{array}$ \\
\hline \multicolumn{4}{|c|}{ Skin and Soft Tissue Infections } \\
\hline Staphylococcus aureus & MSSA - 23\% & MSSA -7.5\% & MSSA -8\% \\
MRSA - 15\% & MRSA - 7.5\% & MRSA - 7\% \\
\hline Pseudomonas aeruginosa & $16 \%$ & $13 \%$ & $15 \%$ \\
\hline E. coli & ESBL-8\% & ESBL-6\% & ESBL-7\% \\
Non ESBL-5\% & Non ESBL -5\% & Non ESBL -4\% \\
\hline Acinetobacter sp. & $9 \%$ & $13 \%$ & $7 \%$ \\
\hline Klebsiella sp. & $9 \%$ & ESBL- 7.2\% & ESBL- 8\% \\
Non ESBL -6 $\%$
\end{tabular}

Staphylococcus aureus, ESBL: Extended Spectrum Beta lactamases

\begin{tabular}{|c|c|c|c|c|c|c|c|c|}
\hline \multirow[t]{2}{*}{ Organism } & \multirow[t]{2}{*}{ Sample } & \multicolumn{7}{|c|}{ Antibiotics* N (\%) } \\
\hline & & & $\mathbf{A k}$ & Cfs & G & Pip/taz & $\mathrm{Mr}$ & I \\
\hline \multirow{5}{*}{ E coli } & \multirow{3}{*}{ Exudate } & $\operatorname{Bef}(n=63)$ & $44(69)$ & $34(53)$ & $29(45)$ & $26(41)$ & $37(58)$ & $37(58)$ \\
\hline & & Aft $(n=247)$ & $216(87)$ & $148(60)$ & $157(64)$ & $132(53)$ & $193(78)$ & $193(73)$ \\
\hline & & \begin{tabular}{|l|}
$\mathrm{p}$ value** \\
\end{tabular} & 0.0017 & & 0.0141 & & 0.0033 & 0.032 \\
\hline & \multirow{2}{*}{ Blood } & $\operatorname{Bef}(n=24)$ & $23(96)$ & $22(92)$ & $14(58)$ & 19(79) & $22(92)$ & $21(88)$ \\
\hline & & Aft(n=33) & $30(91)$ & $25(76)$ & $21(64)$ & $22(67)$ & $27(82)$ & $27(82)$ \\
\hline \multirow{8}{*}{ Klebsiella } & \multirow{3}{*}{ Exudate } & $\operatorname{Bef}(n=112)$ & $50(45)$ & $25(22)$ & $33(29)$ & $23(21)$ & $39(35)$ & $38(34)$ \\
\hline & & $\operatorname{Aft}(\mathrm{n}=303)$ & $184(61)$ & $136(45)$ & $157(51)$ & $124(41)$ & $167(55)$ & $155(51)$ \\
\hline & & p value & 0.0038 & 0.000 & 0.0001 & 0.0001 & 0.0003 & 0.0019 \\
\hline & \multirow{2}{*}{ Blood } & $\operatorname{Bef}(n=26)$ & $22(85)$ & $16(62)$ & $15(58)$ & $14(54)$ & $14(58)$ & $15(54)$ \\
\hline & & Aft(n=33) & $22(67)$ & $16(48)$ & $22(67)$ & $15(45)$ & $20(61)$ & 19(58) \\
\hline & \multirow{3}{*}{ Sputum } & Bef(n=17) & 13(76) & 10(59) & $10(53)$ & $9(53)$ & $10(59)$ & $10(59)$ \\
\hline & & Aft $(n=109)$ & $80(73)$ & $68(62)$ & $66(61)$ & $58(53)$ & $70(62)$ & $73(67)$ \\
\hline & & & Ak & Cfs & Pip/taz & $\mathrm{Mr}$ & I & Ca \\
\hline \multirow{6}{*}{ Pseudomonas } & \multirow{3}{*}{ Exudate } & $\operatorname{Bef}(n=74)$ & $30(41)$ & $32(43)$ & $28(38)$ & $33(45)$ & $32(43)$ & $32(43)$ \\
\hline & & Aft $(n=339)$ & $225(67)$ & $182(54)$ & $171(51)$ & $189(56)$ & $190(57)$ & $197(59)$ \\
\hline & & \begin{tabular}{|l|}
$\mathrm{p}$ value \\
\end{tabular} & 0.0001 & & & & & 0.0208 \\
\hline & \multirow{3}{*}{ Sputum } & $\operatorname{Bef}(n=8)$ & $3(38)$ & $3(38)$ & $2(25)$ & $2(25)$ & $2(25)$ & $3(50)$ \\
\hline & & Aft(n=41) & $39(95)$ & $37(90)$ & $34(82)$ & $31(75)$ & $32(78)$ & $37(90)$ \\
\hline & & p value & 0.0005 & 0.0029 & 0.0025 & 0.0102 & 0.0067 & 0.0029 \\
\hline \multirow{3}{*}{ Acinetobacter } & \multirow{3}{*}{ Exudate } & $\operatorname{Bef}(n=75)$ & $20(27)$ & $18(24)$ & $9(12)$ & $18(24)$ & $12(16)$ & $8(11)$ \\
\hline & & Aft(n=165) & $41(25)$ & 31(19) & $13(14)$ & $24(15)$ & $24(15)$ & $17(10)$ \\
\hline & & & Amc & Cot & G & Clin & Tetra & Lz \\
\hline \multirow{4}{*}{ S. aureus } & \multirow{2}{*}{ Exudate } & $\operatorname{Bef}(n=91)$ & $50(50)$ & 71(78) & $74(81)$ & $70(77)$ & $86(95)$ & $88(97)$ \\
\hline & & $\operatorname{Aft}(\mathrm{n}=332)$ & $180(54)$ & $278(84)$ & $268(81)$ & $259(78)$ & $306(92)$ & $323(87)$ \\
\hline & \multirow{3}{*}{ Blood } & $\operatorname{Bef}(n=23)$ & $13(57)$ & 18(78) & 21(91) & $18(78)$ & $20(90)$ & $22(96)$ \\
\hline & & Aft(n=48) & $25(52)$ & $43(90)$ & $40(83)$ & $37(77)$ & 43(87) & $46(96)$ \\
\hline & & & Pt & Ak & Nf & mc & Cip & Cot \\
\hline \multirow[t]{3}{*}{ E. coli } & Urine & $\operatorname{Bef}(\mathrm{n}=384)$ & $260(68)$ & $347(90)$ & $312(81)$ & $170(44)$ & $123(32)$ & $193(50)$ \\
\hline & & $\operatorname{Aft}(\mathrm{n}=462)$ & $301(65)$ & $414(90)$ & $344(75)$ & $195(42)$ & $139(30)$ & $226(49)$ \\
\hline & & $\mathrm{p}$ value & & & 0.0205 & & & \\
\hline \multirow[t]{2}{*}{ Klebsiella } & Urine & $\operatorname{Bef}(\mathrm{n}=143$ & 73(51) & 94(66) & $25(17)$ & $64(45)$ & $68(48)$ & $81(57)$ \\
\hline & & $\operatorname{Aft}(\mathrm{n}=202)$ & $107(53)$ & $144(72)$ & $48(24)$ & $84(42)$ & $92(49)$ & $112(56)$ \\
\hline \multicolumn{9}{|c|}{$\begin{array}{c}\text { Table 3. Comparison of Antibiotic Susceptibility Patterns before and } \\
\text { after Implementation of Antibiotic Policy }\end{array}$} \\
\hline \multicolumn{9}{|c|}{$\begin{array}{l}\text { Abbrev:-Ak-Amikacin, Cfs- Cefoperazone/sulbactam, G-Gentamicin, Pip/Taz- } \\
\text { Piperacillin/tazobactam, Mr-meropenem, I-Imipenem, Ca-Ceftazidime, Amc- } \\
\text { Amoxicillin/clavulanic acid, Cot-Cotrimoxazole, Clin-Clindamycin, Tetra- } \\
\text { Tetracycline, Lz-Linezolid, Nf-Nitrofurantoin, Cip-Ciprofloxacin, Bef-Before, Aft- } \\
\text { After. } \\
\text { *table does not include all the antibiotics tested, but shows } 6 \text { antibiotics with } \\
\text { highest susceptibility percentage. } \\
{ }_{* *} \text { Only p values which were significant are shown }\end{array}$} \\
\hline
\end{tabular}

\begin{tabular}{|c|c|c|c|c|c|c|}
\hline $\begin{array}{c}\text { MIC } \\
\text { Values }\end{array}$ & $\begin{array}{c}<=2 \mu \mathrm{\mu g} / \mathrm{ml}, \\
\mathbf{N}(\%)\end{array}$ & $\begin{array}{c}\mathbf{4} \boldsymbol{\mu g} / \mathbf{m l} \\
\mathbf{N}(\%)\end{array}$ & $\begin{array}{c}\mathbf{8} \boldsymbol{\mu g} / \mathbf{m l} \\
\mathbf{N}(\%)\end{array}$ & $\begin{array}{c}\mathbf{1 6} \boldsymbol{\mu g} / \mathbf{m l} \\
\mathbf{N}(\%)\end{array}$ & $\begin{array}{c}\mathbf{3 2} \boldsymbol{\mu g} / \mathbf{m l} \\
\mathbf{N}(\%)\end{array}$ & $\begin{array}{c}>=64 \mu \mathrm{g} / \mathbf{m l} \\
\mathbf{N}(\%)\end{array}$ \\
\hline $\begin{array}{c}\text { Before } \\
(\mathrm{n}=63)\end{array}$ & $25(39.7)$ & $8(12.7)$ & $6(9.5)$ & $5(7.9)$ & 0 & $19(30.2)$ \\
\hline $\begin{array}{c}\text { After } \\
(\mathrm{n}=247)\end{array}$ & $145(58.7)$ & $33(13.4)$ & $20(8.1)$ & $16(6.5)$ & $7(2.8)$ & $26(10.6)$ \\
\hline \multicolumn{6}{|c|}{ Table 4. Distribution of Escherichia coli Isolates from Exudate } \\
Samples Based on MIC Values for Amikacin; before and after \\
Implementation of Antibiotic Policy \\
\hline
\end{tabular}




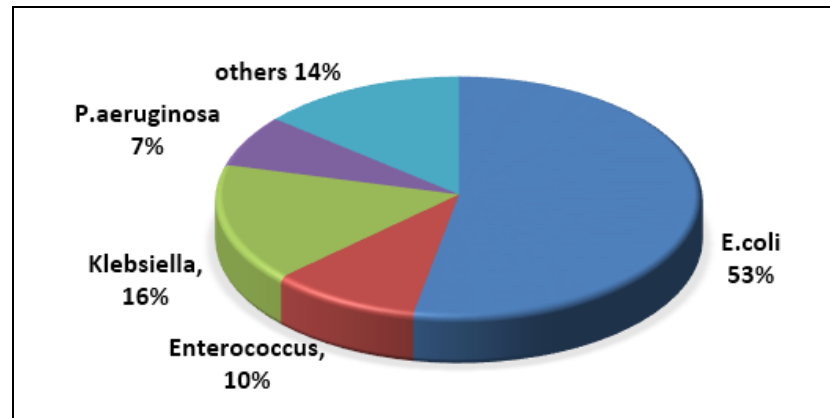

Figure 1. Distribution of Culture Isolates in Urine in 2016-2017

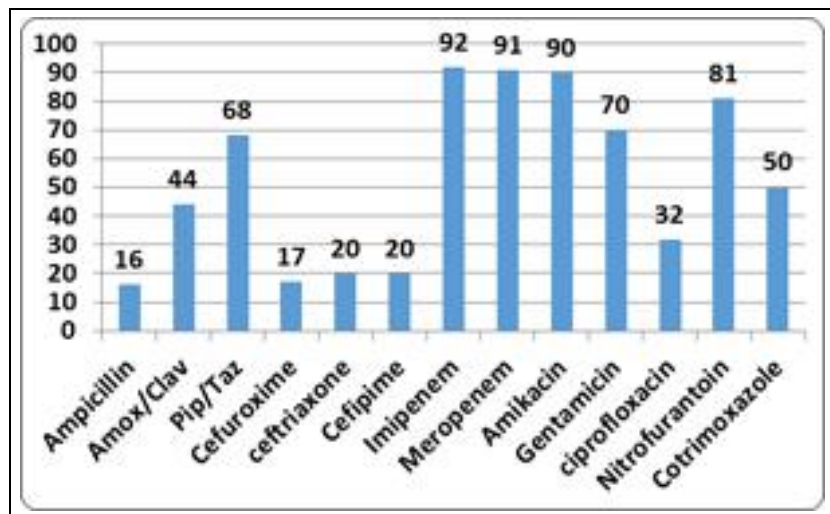

Figure 2. Sensitivity Pattern of Urinary Isolates of Escherichia coli (2016-2017)

Amox/clav: Amoxicillin-Clavulanic acid, Pip/Taz: Piperacillin-Tazobactam

\section{DISCUSSION}

Prompt antimicrobial therapy in case of an infection makes a lot of difference between recovery and death and most of the time prevents long term disability. Unfortunately, the easy availability and familiarity, affordable prices and ignorance of the impending consequences of antimicrobials, has not only resulted in use and misuse of antimicrobials but has also aided the persistent expansion of multidrug resistant microbes, leading to the loss of efficacy of these "magic drugs". Inappropriate antibiotic usage triggers the selection and rapid emergence of drug resistant bacteria like ESBLs, which in turn spread in the community by horizontal gene transfer. ${ }^{8}$

In 2013, The Indian Council for Medical Research (ICMR) has started an antimicrobial resistance surveillance and research network. Now it involves 6 nodal centers, 16 regional centers to monitor trends in the antimicrobial profile in the country. ${ }^{9}$ According to ICMR data of 2017, in India gram negative pathogens are dominant. $95 \%$ of respiratory isolates, $81 \%$ of urine isolates and $60 \%$ of blood isolates are gram negative bacteria. ${ }^{10}$ In our study, an average of $78 \%$ of urinary infections is caused by gram negative bacteria namely; Escherichia coli, Klebsiella species and Pseudomonas aeruginosa and $77 \%$ of respiratory isolates included Klebsiella species, Acinetobacter species and Pseudomonas aeruginosa.

In our study we have demonstrated a decrease in the infection rate due to drug resistant organisms like MRSA and ESBL; and an increase in the susceptibility to commonly used antibiotics following adherence to institution antibiotic protocol. Prevalence of Staphylococcus aureus in skin and soft tissue infections reduced from 38\% (2016-2017) to 15\% (2017-2018) with MRSA rates decreasing from 15\% to $7.5 \%$. ICMR found that there was marginal reduction in the infections caused by MRSA with $42.6 \%$ in 2015 to $33.78 \%$ in 2016-2017.9 Thus, antibiogram helps to improve awareness and understanding of the local trends in antimicrobial resistance. Each health care facility should be aware of their antimicrobial resistance patterns; which can pave the way to develop infection control protocols and antimicrobial stewardship programs. Effective infection control program brings down the hospital acquired infection rates. This in turn decreases the amount of antibiotic consumption and helps to contain antimicrobial resistance. National Action Plan on Antimicrobial resistance (NAP-AMR) has outlined 6 strategic plans to be implemented over 2017-2021. The fourth strategy includes development of antimicrobial stewardship in healthcare. 11

Antibiotic stewardship refers to "coordinated interventions designed to improve and measure the appropriate use of antimicrobial agents by promoting the selection of the optimal antimicrobial drug regimen including dosing, duration of therapy, and route of administration".12 This can be done by framing and implementation of infectious disease treatment guidelines, regular audits and providing feedback to higher authorities. ${ }^{13}$ The first ever meeting of medical societies in India was held in the $2^{\text {nd }}$ annual conference of Clinical Infectious Disease Society of India (CISCON) at Chennai in 2012; which lead to the Chennai Declaration. Formulating plans to tackle the problem of AMR at the national level was its main intention. It includes description of the roles and responsibilities of hospitals and microbiology laboratories in the fight against AMR. All hospitals should have an active Hospital Infection Control committee which should guide in initiation or augment the efforts in antibiotic policy implementation. It suggests various steps for conversion of a conventional microbiologist to an active player in prevention of hospital acquired infections, to derive and implement antibiotic policy, monitoring AMR and strengthen diagnostic facility. ${ }^{14}$

A good surveillance system is necessary for an effective antimicrobial stewardship program. The system should be equipped to detect significant differences, identify changes in sensitivity patterns of various organisms and promptly report the information derived from them. 15 "WHONET" is the software program which can be used for the analysis of data from different laboratories. ${ }^{16}$

Preparation and annual updating of local antibiogram with susceptibility data of specific pathogens is recommended in order to optimize empiric antibiotic therapy. Our study showed slight increase in susceptibility of various pathogens to various antibiotics. Monitoring yearly trends and comparison of data, and review in the antibiotic committee helps in deciding changes needed in the existing antibiotic protocol. The cumulative antibiogram pattern in our study shows the local epidemiology and susceptible patterns may not be applicable to other geographic regions. In addition, factors like sampling methodology, patient demographics and study setting may further hamper comparability of our results to those of others. 
In our hospital, good compliance for the antibiotic policy could be due to the flexible general rules for antibiotic prescribing. The Chennai declaration also opined for a step by step implementation of a national antibiotic policy rather than a strict policy in a country like India where antibiotic usage is so rampant.14 Complete adherence to a hospital antibiotic policy may not be possible. Pressure from patients sometimes forces medical practitioners to overprescribe antibiotics. In addition to in-vitro susceptibility of a bacterium to an antibiotic, factors like cost effectiveness, adverse effects, selection of resistant strains have to be considered to maintain the efficacy and safety of the treatment given to a patient. ${ }^{17}$ This may be reflected in the compliance rates for an antibiotic policy.

A study by Chandy SJ et al showed that the development of antibiotic policy guidelines and its dissemination contributed significantly to containing antibiotic use. The study also showed that the mode of policy implementation was critical for the effective usage of the policy. Use of computer network for online intranet access to policy guidelines showed a significant decrease in antibiotic use. ${ }^{18}$

\section{CONCLUSIONS}

Antimicrobial resistance is a major issue in India. Of late, there have been efforts to combat this problem at the national level. A rapid and robust system to implement the policies formulated at the national level is vital. In every healthcare setup, antimicrobial policy should be one of the mandatory requirements, and making antibiogram is the first step before framing antibiotic policy. Hospital infections and increasing incidence of antimicrobial resistance prolongs hospital stay and enhances economic burden of patient. Acceptance and compliance by clinicians are essential for successful implementation of the policy. Strict adherence to the infection control practices and judicious use of antibiotics remains the only way to counter the threat of antimicrobial resistance.

\section{REFERENCES}

[1] Antimicrobial resistance: tackling a crisis for the health and wealth of nations. Review on antimicrobial resistance. 2014.2 https://www.amrreview.org/sites/default/files/AMR\%20Review\%20

Paper\%20\%20Tackling\%20a\%20crisis\%20for\%20\%3C sup $\% 3 \mathrm{Eth} \% 3 \mathrm{C} /$ sup $\% 3 \mathrm{E} \% 20 \mathrm{e} \% 20$ health $\% 20$ and $\% 20 \mathrm{w}$ ealth\%20of\%20nations_1.pdf [Last accessed on 2019 Oct 23].

[2] Nordmann P, Naas T, Poirel L. Global spread of carbapenemase-producing Enterobacteriaceae. Emerg Infect Dis 2011;17(10):1791-8.

[3] Combating Antimicrobial Resistance in India. http://www.searo.who.int/india/topics/antimicrobial_r esistance/Combating_Antimicrobial_Resistance_in_India /en/ [last accessed on 2019 Oct 23].

[4] Hindler JF, Stelling J. Analysis and presentation of cumulative antibiograms: a new consensus guideline from the Clinical and Laboratory Standards Institute. Clin Infect Dis 2007;44(6):867-73.

[5] Kohlmann R, Gatermann SG. Analysis and presentation of cumulative antimicrobial susceptibility test data- the influence of different parameters in a routine clinical microbiology laboratory. PLoS One 2016;11(1):e0147965.

[6] CLSI. Analysis and presentation of cumulative antimicrobial susceptibility test data: approved guideline. CLSI document M39-A4. 4th edn. Wayne, PA: Clinical and Laboratory Standards Institute 2014.

[7] National treatment guidelines for Antimicrobial Use in Infectious Diseases. National Centre for Disease Control, Directorate General of Health Services, Ministry of Health and Family Welfare, Government of India. Version 1.0. 2016:1-64 http://pbhealth.gov.in/AMR_guideline7001495889.pdf [last accessed on 2019 Oct 10].

[8] Hawkey PM, Jones AM. The changing epidemiology of resistance. Journal of Antimicrobial Chemotherapy 2009;64(Suppl 1):i3-i10.

[9] Walia K, Madhumati J, Veeraraghavan B, et al. Establishing antimicrobial resistance surveillance \& research network in India: journey so far. Indian J Med Res 2019;149(2):164-79.

[10] Walia K, Ohri VC, Singh H. Annual Report Antimicrobial Resistance Surveillance Network January 2017December 2017. AMR Surveillance Network Indian Council of Medical Research 2017:1-51.

[11] National Action Plan on Antimicrobial Resistance. Ministry of Health and Family Welfare, Government of India 2017:1-53. www.ncdc.gov.in. [Last accessed on 2019 Oct 10].

[12] Fishman N, Patterson J, Saiman L, et al. Policy statement on antimicrobial stewardship by the Society for Healthcare Epidemiology of America (SHEA), the Infectious Diseases Society of America (IDSA), and the Paediatric Infectious Diseases Society (PIDS). Infect Control Hosp Epidemiol 2012;33(4):322-7.

[13] Ranjalkar J, Chandy SJ. India's National Action Plan for antimicrobial resistance - an overview of the context, status and way ahead. J Family Med Prim Care 2019;8(6):1828-34.

[14] Ghafur A, Mathai D, Muruganathan A, et al. The Chennai Declaration: a roadmap to tackle the challenge of antimicrobial resistance. Indian Journal of Cancer 2012;49(4):84-94.

[15] Bax R, Bywater R, Cornaglia G, et al. Surveillance of antimicrobial resistance-what, how and whither? Clin Microbiol Infect 2001;7(6):316-25

[16] Stelling JM, O'Brien TF. Surveillance of antimicrobial resistance: the WHONET Program. Clin Infect Dis 1997;24(Suppl 1):S157-S68.

[17] Gupta K, Hooton TM, Stamm WE. Increasing antimicrobial resistance and the management of uncomplicated community-acquired urinary tract infections. Ann Intern Med 2001;135(1):41-50.

[18] Chandy SJ, Naik GS, Charles R, et al. The impact of policy guidelines on hospital antibiotic use over a decade: a segmented time series analysis. PLoS One 2014;9(3):e92206. 